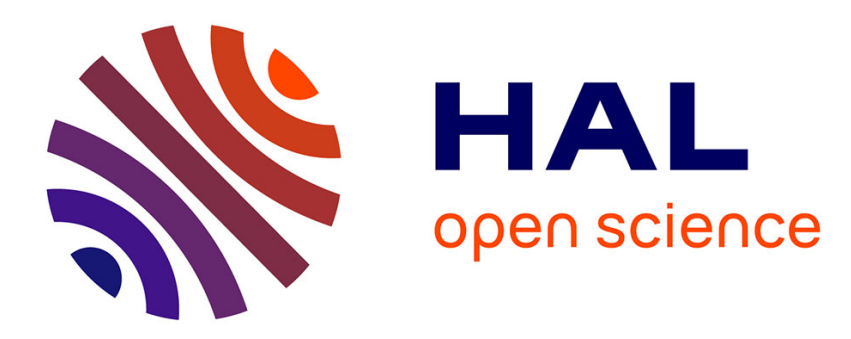

\title{
Wetting Behaviour of Thin Diblock Films
}

Matthew Turner, A. Johner, J.-F. Joanny

\section{To cite this version:}

Matthew Turner, A. Johner, J.-F. Joanny. Wetting Behaviour of Thin Diblock Films. Journal de Physique I, 1995, 5 (7), pp.917-932. 10.1051/jp1:1995102 • jpa-00247110

\section{HAL Id: jpa-00247110 https://hal.science/jpa-00247110}

Submitted on 1 Jan 1995

HAL is a multi-disciplinary open access archive for the deposit and dissemination of scientific research documents, whether they are published or not. The documents may come from teaching and research institutions in France or abroad, or from public or private research centers.
L'archive ouverte pluridisciplinaire HAL, est destinée au dépôt et à la diffusion de documents scientifiques de niveau recherche, publiés ou non, émanant des établissements d'enseignement et de recherche français ou étrangers, des laboratoires publics ou privés. 
Classification

Physics Abstracts

$68.45 \mathrm{Gd}-68.10 \mathrm{Cr}-83.70 \mathrm{Hq}$

\title{
Wetting Behaviour of Thin Diblock Films
}

\author{
Matthew S. Turner $\left({ }^{1, *}\right)$, A. Johner $\left({ }^{2}\right)$ and J.-F. Joanny $\left({ }^{2}\right)$ \\ ( $\left.{ }^{1}\right)$ Cavendish Laboratory, Madingley Road, Cambridge CB3 OHE, UK \\ $\left({ }^{2}\right)$ Institut Charles Sadron, CRM, 6 rue Boussingault, 67083 Strasbourg Cedex, France \\ (Received 5 December 1994, received in final form 9 March 1995, accepted 15 March 1995)
}

\begin{abstract}
We study the wetting behaviour of a thin diblock copolymer film deposited on a solid substrate. Near the wetting edge of a monolayer we find that the chains may be oriented parallel to the surface, whereas in the layer interior they are perpendicular to it. These two orientations give rise to two wetting transitions. Various edge configurations are predicted, depending on the surface tensions. We find that bending/splay terms are only ever important near the wetting edge where they fix the chain orrentation parallel to the wetting edge. We also estimate the line tension associated with the wetting edge. This quantity will control the growth of droplets.
\end{abstract}

\section{Introduction}

The equilibrium properties of diblock copolymers have been of increasing interest to theoreticians $[1,2]$ and experimentalists [3-9] over the last few years. It is now well-known that, for temperatures below the order-disorder transition (ODT), molten diblock copolymers selfassemble spontaneously to form ordered bulk phases consisting of sheets (the lamellar phase), rods (the hexagonal phase), spheres (the cubic phase) or even multi-connected, bicontinuous phases. This self-assembly is driven by the immiscibility of the two different chemical components of the polymer, denoted $\mathrm{A}$ and $\mathrm{B}$. By forming a segregated phase the system minimises the number of A-B contacts, thereby saving energy which offsets the corresponding loss of polymer entropy. In the so-called weak segregation limit the excess of one of the species varies smoothly over the sample, whilst in the strong segregation limit, well below the ODT, there is a sharp interface between alternate regions of almost pure A or B blocks. Reference [10] provides a good review of this field. Recently, much experimental work has focused on the properties of thin copolymer films [5-9,11,12]. Whilst some theoretical [13-15] and computational [16] work does exist, little is known about the detailed wetting properties of such films. In the present work we aim to construct such a theory, focussing on the strong segregation limit.

The wetting of a surface by a layer of simple liquid is a problem which has been of continuing interest to scientists from the beginning of the last century, with considerable recent

$\left(^{*}\right)$ Author to whom correspondence should be addressed 


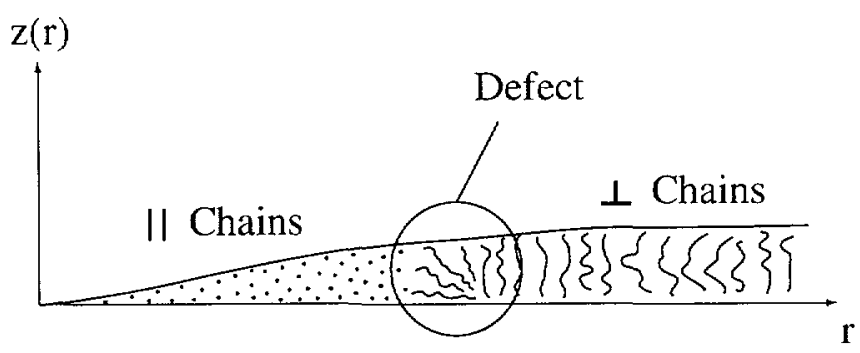

Fig. 1. - Sketch showing the structure of a wetting monolayer of copolymers for appropriately chosen parameter values $\left(\bar{\gamma}_{A B}<1\right.$ and $0<\bar{\gamma}_{\| I}<1$, see Section 2 below). $z$ and $r$ are dimensionless, in units of $h_{\infty}$ the equilibrium monolayer thickness (at $r \rightarrow \infty$ ). In this case the chains are $\|$ to the substrate surface and parallel to the wetting edge (into the page) near $r=0$. There is a defect near $r=r^{*}$, where the $\|$ and $\perp$ regions meet.

attention being given to the interesting problem of liquid spreading [17]. The study of wetting has obvious important applications in, e.g., paints, gluing, lubrication, etc. In simple liquids there are certain long-range forces present but the liquid itself has no intrinsic ordering. Such ordering is present in diblock copolymer melts which can be expected to exhibit interesting non-classical wetting behaviour. Indeed, as we shall see below, diblock copolymers exhibit two wetting transitions. This is related to their ability to align roughly parallel or perpendicular to the substrate surface. In marked contrast to short rigid molecules, a single diblock copolymer lamella (a monolayer) can switch from its equilibrium perpendicular ordering to parallel ordering when confined between two parallel plates as their seperation is altered (see Appendix A). In the following we will argue that a qualitatively similar transition may take place in the wetting edge of a lamella deposited on a solid substrate, the regions of perpendicular and parallel ordering being separated by a localized "defect".

We consider a wetting layer of monodisperse A-B diblock copolymers on a flat substrate in contact with air. Confinement between two plates is a qualitatively different problem and is discussed only in Appendix A. For large droplets we may reduce the problem to two dimensions, where $x$ is the coordinate along the substrate and $h(x)$ is the perpendicular distance from the substrate to the copolymer-air interface. For definiteness the edge of the copolymer layer, where the copolymer, substrate and air meet defines $x=0$; the copolymer layer is found for $x>0$. We adopt units where the statistical segment length $b=1$ and $k_{\mathrm{B}} T=1$. We also assume throughout that the chains remain strongly ordered (1.e., we are well below the ODT) and that the effect of free volume is negligible. These approximations are appropriate in the (conventional) $N \rightarrow \infty$ limit for finite A-B and air-polymer $\chi$-parameters and will be discussed further in Section 6 below.

In order to determine the internal structure of the layer we must compare the free energies of two possible chain configurations: (i) Where the average chain end-to-end vector is approximately perpendicular to the substrate, hereafter referred to as the perpendicular $\perp$ configuration, and (ii) where the average chain end-to-end vector is approximately parallel to the substrate, the parallel $\|$ configuration. Under certain circumstances, discussed below, the structure of the layer may resemble Figure 1. Some earlier experimental studies [8,9] have suggested the existence of "mixed" layers which may be regions of $\|$ ordering. Very recent experiments [12] provide direct evidence for a region of $\|$ ordering near the edge of a wetting layer.

We unitially restrict our attention to the case where an $\mathrm{AB}$ "monolayer" is the equilibrium 
state, not an ABBA or BAAB "bilayer". The conditions under which this assumption is valid are briefly discussed in Section 5 below, as is the opposite case where the bilayer is more stable [13]. Note that we need only consider these two cases (monolayer and bilayer) as it can be shown that either (a) one of these two configurations is the minimum energy state for $\perp$ ordering, or (b) the $\perp$ chains de-wet the surface, forming droplets. This applies only in the absence of additional constraints, such as fixed substrate area or film thickness (see Appendix A and Ref. [14]). Case (b), as well as some aspects of case (a), were discussed, for flat equilibrium layers, in an earlier article [13].

We may summarise the behaviour as follows: either single or double layers or droplet(s) are found, whichever corresponds to the configuration with the lowest total interfacial tension per chain. These conditions are discussed in Sections 3 and 5.

In the monolayer (or $\perp$ region) we label the blocks which reside at the substrate and at the air interface $A$ and $B$, respectively. We will assume that a continuous representation of the layer is appropriate. This assumption fails when, e.g., thickness of the 1 region becomes comparable to a molecular length.

\section{Free Energies}

The dimensionless free energy density for the copolymer layer takes different forms depending on whether the chains are $\perp$ or $\|$ to the substrate. However, in both cases we include four terms (i) the contribution from the entropy of chain stretching, (ii) the surface energies, parameterised by the surface tension at each interface, (iii) the bending contribution due to the splay between neighbouring chains, and (iv) Van-der-Waals interactions, the source of the so-called disjoining pressure [17]. We will find later that these last two terms only become significant very near the layer edge. The bending contribution for $\|$ chains is also dependant on the orientation of the chains in the plane of the surface: (i) If the chains have an end-to-end vector perpendicular to the wetting edge, denoted $11 . \nabla$, there is a finite bending energy, significant very near the layer edge (see Appendix B), but if (ii) the end-to-end vectors are parallel to the wetting edge, denoted simply $\|$, there is no bending contribution at the level of our treatment. This will cause the chains to lie in the simple $\|$ orientation. However, for completeness, we give some results in Appendix B for the case where the chains are artificially constrained to lie in the $\|, \nabla$ direction. This might be relevant if there is some anchoring of the orientation at either the substrate or the defect.

2.1. The Energy of Chains $\perp$ to the Substrate. - For chains $\perp$ to the substrate the dimensionless free energy per unit volume, $F_{\perp}$, is constructed from the four terms mentioned above: (i) The elastic energy of each polymer chain $\frac{k}{2 N} h^{2}$, where $N$ is the number of monomers per chain and $k$ is a constant of order one, gives a free energy per unit volume $\frac{k}{2 N^{2}} h^{2}$. The surface energy per unit volume $\frac{1}{h}\left(\gamma_{\hat{A S}}+\gamma_{\mathrm{AB}}+\gamma_{\mathrm{B} \text { arr }}\left[1+\frac{1}{2}\left(\frac{\mathrm{d} h}{\mathrm{~d} x}\right)^{2}\right]\right)$ includes the contribution from the three interfaces, where $\gamma_{A B}$ and $\gamma_{B \text { arr }}$ are the dimensionless surface tensions acting at the A-B and B-air interfaces respectively and $\gamma_{\hat{A S S}} \equiv \gamma_{\mathrm{AS}}-\gamma_{\mathrm{S} \text { ar }}$ is the nett surface tension acting at the A-surface interface. Note that we have included the leading order term in a gradient expansion at the polymer-air interface. (iii) The bending contribution $\frac{\kappa_{\perp}}{2}\left(\frac{\mathrm{d}^{2} h}{\mathrm{~d} x^{2}}\right)^{2}$ to leading order, scaling like the squared angle between the end-to-end vector of neighbouring 
chains $\theta \simeq \frac{\mathrm{d}^{2} h}{\mathrm{~d} x^{2}}$. (iv) Finally there are the Van-der-Waals interactions giving an energy contribution $H /\left(12 \pi h^{3}\right)$ per unit volume, with $H$ the Hamacker constant. Collecting these four terms we have

$$
F_{\perp}=\frac{k}{2 N^{2}} h^{2}+\frac{1}{h}\left(\gamma_{\hat{A S}}+\gamma_{\mathrm{AB}}+\gamma_{\mathrm{B} \text { aIr }}+\frac{\gamma_{\mathrm{B} \cdot \mathrm{aIr}}}{2}\left(\frac{\mathrm{d} h}{\mathrm{~d} x}\right)^{2}\right)+\frac{\kappa_{\perp}}{2}\left(\frac{\mathrm{d}^{2} h}{\mathrm{~d} x^{2}}\right)^{2}+\frac{H}{12 \pi h^{3}}
$$

It is known [18] that the bending modulus for copolymer lamellar layers in the bulk is $\simeq \gamma_{\mathrm{AB}} h$, this being the only quantity with the correct dimensionality that can be constructed from the two intrinsic scales $\gamma_{\mathrm{AB}}$ and $h$. In what follows we will estimate the modulus $\kappa_{\perp}$ according to $\kappa_{\perp} \simeq \gamma_{\mathrm{AB}} h$, which should be a reasonable approximation provided $h$ is slowly varying. In fact we will see that the bending energy is anyway negligible in the region of $\perp$ chains [19].

By rescaling equation (1) with respect to the characteristic length $h_{\infty}=\left(\tilde{\gamma} N^{2} / k\right)^{1 / 3}$, the value of $h$ which minımises $F_{\perp}$ with $\tilde{\gamma}=\gamma_{\mathrm{AB}}+\gamma_{\mathrm{AS}}+\gamma_{\mathrm{B} \text { arr }}$, this equation simplifies to

$$
F_{\perp}=\mu\left\{\frac{z^{2}}{3}+\frac{2}{3 z}\left(1+\frac{\bar{\gamma}_{\mathrm{Ba}}}{2}\left(\frac{\mathrm{d} z}{\mathrm{~d} r}\right)^{2}\right)+\frac{\bar{K}_{\perp} z}{2}\left(\frac{\mathrm{d}^{2} z}{\mathrm{~d} r^{2}}\right)^{2}+\frac{A}{12 \pi z^{3}}\right\}
$$

where $z=h / h_{\infty}, r=x / h_{\infty}, A=\frac{H}{\mu h_{\infty}^{3}}$

$$
\mu=\frac{3}{2}\left(\frac{k \tilde{\gamma}^{2}}{N^{2}}\right)^{1 / 3}
$$

and throughout $\mathrm{a}^{-}$(bar) denotes division by $\tilde{\gamma}$, e.g., $\bar{\gamma}_{\mathrm{B} \text { arr }}=\gamma_{\mathrm{B} \cdot \mathrm{arr}} / \tilde{\gamma}$. The natural bending modulus in these units is $\bar{K}_{\perp} \equiv \frac{\kappa_{\perp}}{h \tilde{\gamma}} \simeq \bar{\gamma}_{A B}$ and the energy density of a (flat) equilibrium $\perp$ monolayer, or the chains at $r \rightarrow \infty$ in the wetting layer, is $\mu$.

2.2. The Energy of Chains " To The Substrate. - For chains $\|$ to the substrate the dimensionless free energy density $F_{\|}$is constructed from the sum of: (i) The elastic energy density $\frac{k}{2 N^{2}} y^{2}$, where $y$ is the end-to-end distance of a $\|$ chain. (ii) The free energy density due to the A-B interface is $\gamma_{\mathrm{AB}} / y$ while the other two interfaces contribute $\frac{1}{h}\left(\gamma_{\| \cdot \mathrm{S}}+\gamma_{\| \cdot \text { air }}\left[1+\frac{1}{2}\left(\frac{\mathrm{d} h}{\mathrm{~d} x}\right)^{2}\right]\right)$, where $\gamma_{\| \cdot \text { air }}$ is the surface tension acting at the $\|$-air interface and $\gamma_{\| \cdot \mathrm{S}} \equiv \gamma_{\| \cdot \mathrm{S}}-\gamma_{\mathrm{s} \cdot \mathrm{ar}}$ is the nett surface tension acting at the $\|$-surface interface. (iii) Finally there are the Van-der-Waals interactions $H /\left(12 \pi h^{3}\right)$. As discussed briefly above, and in Appendix $B$, there is no bending contribution to the free energy provided the end-to-end vectors of the chains lie parallel to the wetting edge. To see this notice that in the only other orthogonal $\| \nabla$ orientation there exists a finite angle between the end-to-end vectors of neighbouring chains $\theta \simeq \frac{1}{h} \frac{\mathrm{d} h}{\mathrm{~d} x}$ but there is no such term in the simple " orientation. In what follows we therefore restrict our attention to simple $\|$ orientation.

Combining these terms we obtain

$$
F_{\|}=\frac{k}{2 N^{2}} y^{2}+\frac{\gamma_{\mathrm{AB}}}{y}+\frac{1}{h}\left(\gamma_{\| \cdot \mathrm{S}}+\gamma_{\| 1 \cdot \mathrm{alr}}+\frac{\gamma_{\| \cdot \mathrm{air}}}{2}\left(\frac{\mathrm{d} h}{\mathrm{~d} x}\right)^{2}\right)+\frac{H}{12 \pi h^{3}}
$$


By minimising equation (4) with respect to $y$ and rescaling according to $z=h / h_{\infty}, r=x / h_{\infty}$ with $h_{\infty}=\left(\tilde{\gamma} N^{2} / k\right)^{1 / 3}$ (as before) we have

$$
F_{\|}=\mu\left\{\bar{\gamma}_{\mathrm{AB}}^{2 / 3}+\frac{2}{3 z}\left(\bar{\gamma}_{\|}+\frac{\bar{\gamma}_{\| \cdot \mathrm{air}}}{2}\left(\frac{\mathrm{d} z}{\mathrm{~d} r}\right)^{2}\right)+\frac{A}{12 \pi z^{3}}\right\}
$$

where $\bar{\gamma}_{\| 1}=\bar{\gamma}_{1 \mid \hat{\text { S }}}+\bar{\gamma}_{\| 1 \cdot \text { air }}$ and we recall that $\mathrm{a}^{-}$(bar) denotes division by $\tilde{\gamma}=\gamma_{\mathrm{AB}}+\gamma_{\mathrm{AS}}+\gamma_{\mathrm{B} \cdot \mathrm{air}}$, e.g., $\bar{\gamma}_{11 \cdot \text { arr }}=\gamma_{11 \cdot \text { air }} / \tilde{\gamma}$.

\section{Two Wetting Transitions}

We can make several important observations by inspection of equations (2) and (5). These depend on whether $\bar{\gamma}_{\|}=\bar{\gamma}_{\| \text {.S }}+\bar{\gamma}_{\| \text {.arr }}$ and $\bar{\gamma}_{\|}-1$ are positive or negative. We will see that $\bar{\gamma}_{\|}=0$ corresponds to the wetting transition for $\|$ chains. There is also a wetting transition for the $\perp$ chains, which is found at $\bar{\gamma}_{A B}=1$. To understand how there can be two wetting transitions for the same copolymer it is important to bear in mind that the copolymer can reside in two distinct states, $\perp$ and $\|$ to the substrate. The wetting transition for the $\perp$ chains occurs when they can obtain a lower energy by coating the substrate with a monolayer than reverting to the bulk. Similarly the $1 /$ wetting transition occurs when energy is gained by covernng bare surface with $\|$ chains.

(i) $\bar{\gamma}_{11}>0$

For this case we may compare the minima of $F_{\perp}($ at $z=1)$ and $F_{\|}$(at $\left.z \rightarrow \infty\right)$ to see that a wetting layer of $\perp$ region exists if, and only if, $\bar{\gamma}_{\mathrm{AB}}>1$, i.e., $\gamma_{\mathrm{AS}}+\gamma_{\mathrm{B} \text {.arr }}<0$. If instead $\bar{\gamma}_{\mathrm{AB}}<1$ the $\perp$ chains de-wet the surface, obtaining the lower (bulk) free energy density $F_{\|}(z \rightarrow \infty)$. Note that the wetting transition for $\perp$ chains can be identified by comparing the total surface tension acting on a wetting layer of $\perp$ chains $\tilde{\gamma}=\gamma_{A B}+\gamma_{A S}+\gamma_{B \text { air }}$ with the surface tension acting on a layer in the bulk $\gamma_{\mathrm{AB}}$. The wetting layer is stable only when $\tilde{\gamma}<\gamma_{\mathrm{AB}}$, i.e., $\bar{\gamma}_{\mathrm{AB}}>1$.

(i) $\vec{\gamma}_{\|}<0$

We next imagine varying $\bar{\gamma}_{\|}$through zero to a negative value. At $\bar{\gamma}_{\|}=0$ there is a wetting transition for the $1 /$ chains: Energy is gained proportional to the area of bare surface covered by these chains. This transition may be identified if we consider the forces acting at the point (line) where the surface, copolymer and air meet.

The behaviour for $\bar{\gamma}_{\|}<0$ can be discussed within the assumption that, at the wetting transition, all available bare surface is covered by a very thin layer of $\|$ chains. The minimum thickness of this layer is limited by the disjoining pressure (Van-der-Waals forces) to the order of a molecular length [17], which is assumed to be much smaller than any other length scale that appears in the problem. However so-called free volume effects may also provide a limiting thickness [20]. In either case, as a first approxımation, we might proceed by re-normalising two of the surface tensions according to

$$
\gamma_{\| \hat{I} \cdot \mathrm{S}}^{\prime}=0 \quad \gamma_{\hat{A} \hat{S}}^{\prime}=\gamma_{\hat{A} \hat{S}}-\gamma_{\| \hat{I} \cdot \mathrm{S}}
$$

noting that a re-normalisation of $\gamma_{\hat{A} \mathbf{S}}$ implies a re-normalisation of $\tilde{\gamma}$ and consequently all parameters scaled by $\tilde{\gamma}$, i.e., all parameters with $\mathrm{a}^{-}$(bar). In particular this results in a renormalised $\bar{\gamma}_{\| l}^{\prime}$, for which $\bar{\gamma}_{\|}^{\prime}>0$, and a renormalised $\bar{\gamma}_{A B}^{\prime}$, which may or may not satisfy $\bar{\gamma}_{A B}^{\prime}>1$. Since $\bar{\gamma}_{\| 1}^{\prime}>0$ the discussion of part (i) above again applies on replacing all the parameters with their new (primed) versions. Hence if $\bar{\gamma}_{\mathrm{AB}}^{\prime}<1$ all chains (except those involved in the very thin wetting layer) de-wet and revert to the bulk. Whereas for $\bar{\gamma}_{\mathrm{AB}}^{\prime}>1$ we retain a wetting layer of $\perp$ chains, as shown in Figure 1 , with the single modification that there 
is no bare surface; it is now covered by a very thin layer of $1 /$ chains. Finally we remark that any properties depending on those surface tensions which are renormalised, e.g., the surface contour of the polymer film, described by $z(r)$, will exhibit discontinuous behaviour across the wetting transition at $\bar{\gamma}_{\mid 1}=0$.

By examining the energies as $z \rightarrow 0$ we see that $\|$ chains exist near $r=0$ if and only if $\bar{\gamma}_{\|}<1$, otherwise there are only $\perp$ chains. Where we discuss $\|$ ordering in what follows we implicitly assume $0<\bar{\gamma}_{\|}<1$.

3.1. The Surface Contour Near the Edge of the Wetting Layer. - We are now in a position to construct the total free energy $F_{\text {TOT }}$ of the layer as the sum of the contributions from the $\perp$ and $\|$ regions. We will then be able to minimise to determine the size of the " region and the full surface contour $z(r)$. Our approach involves two implicit assumptions: (i) That a continuous representation of the layer is appropriate. This assumption may fail when the lateral extent of the $\|$ region is not greater than the end-to-end distance of the chains, as well as when its thickness becomes comparable to a monomer length. (ii) That the effect of the defect, which occurs at the interface between the $\|$ and $\perp$ regions, may be neglected. This assumption will not affect our discussion of either the two wetting transitions (see Section 3 above) or the scaling results for the size of the II region derived below. In addition we assume that the defect will be rather localised and will have a characteristic energy scale proportional to $\gamma_{A B}$, which in turn is typically much less than the surface tension acting at the copolymer-air interface: For copolymer in contact with aur typically $\gamma_{\mathrm{AB}} / \gamma_{\mathrm{B} \text { air }} \lesssim 1 / 10$. Formally this means that we can neglect the effect of the defect on the same grounds that we neglect the bending contribution to the free energy in the discussion below.

$F_{\text {тот }}$ is given by

$$
F_{\mathrm{TOT}}=\int_{0}^{r^{*}}\left(F_{\|}-\mu\right) z \mathrm{~d} r+\int_{r^{*}}^{\infty}\left(F_{\perp}-\mu\right) z \mathrm{~d} r
$$

where $\mu$ here acts as the chemical potential for exchange of material with the edge (near $r=0$ ) and interior $(r \rightarrow \infty)$ of the layer. Since we have neglected the effect of the defect (interface) between the $\|$ and the $\perp$ regions we lose the exact coupling between the two regions, which are found for $r<r^{*}$ and $r>r^{*}$ respectively, and we must minimise the two integrals in equation (7) separately. By imposing continuity of $z(r)$ at $r^{*}$, we hope to obtain the correct profile $z(r)$ at scales larger than the defect.

We consider first the integral $\int_{0}^{r^{*}}\left(F_{\|}-\mu\right) z \mathrm{~d} z$. Direct minimisation of this is straightforward, we obtain the slope of the profile as a function of the profile, setting $g(z) \equiv(\mathrm{d} z / \mathrm{d} r)^{2}$.

$$
g(z)=\frac{m z+A /\left(4 \pi z^{2}\right)+C}{\bar{\gamma}_{\| \cdot \mathrm{alr}}}
$$

where the definition $m \equiv 3\left(\bar{\gamma}_{\mathrm{AB}}^{2 / 3}-1\right)$ simplifies the notation and the integration constant $C$ is determined by requiring Young's law to be satisfied for the slope of the macroscopic profile (the forces must balance). In the limit where the Van-der-Waals contribution is absent $(A \rightarrow 0)$ or, equivalently, if we examine the contour just outside the region where it is significant $C$ is given by

$$
\frac{C}{\bar{\gamma}_{\| \cdot \text { aur }}}=\left(\gamma_{\| \cdot \text { aur }} / \gamma_{\| \cdot \text { S S }}\right)^{2}-1
$$

Equation (8) 1s one of the main results of this paper. It describes the microscopic tip and is used to obtain details of the profile close to the edge, as presented in Figure 2. In the following 


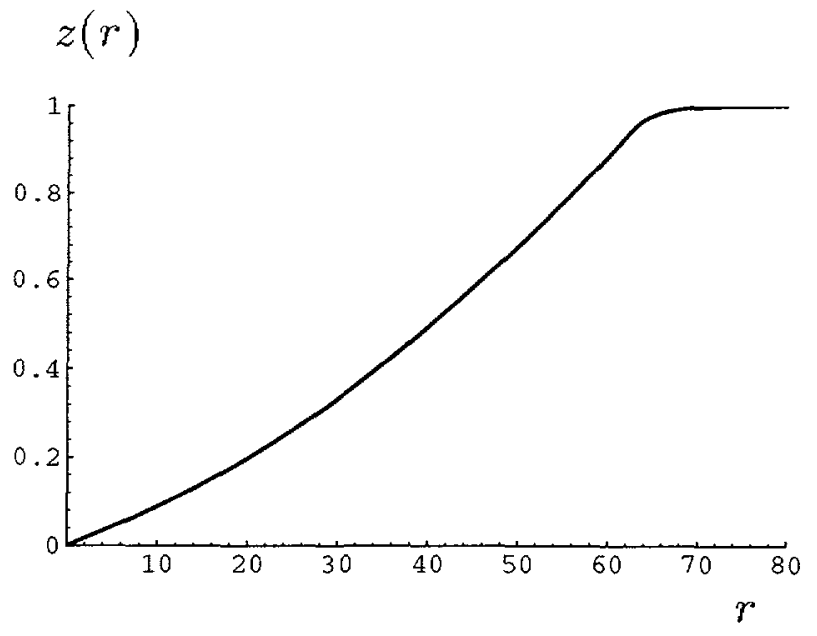

Fig. 2. - Plot of the profile of the wetting layer according to equations (8) and (13) for the following parameter values: $A=8 \pi 10^{-8}, m \equiv 3\left(\bar{\gamma}_{\mathrm{AB}}^{2 / 3}-1\right)=0.01, C=0.001, \bar{\gamma}_{11 \text { air }}=\bar{\gamma}_{\mathrm{B} \text { air }}=20$. Notice that the horizontal scale is much larger than the vertical. For these values, the crossover between 11 and $\perp$ ordering occurs at $z^{*}=0.94, r^{*}=62$ according to equation (14). (The approximate values obtained from Eq. (15) differ only at the $8^{\text {th }}$ and $6^{\text {th }}$ decimal places, respectively). On this scale the Van-der-Waals regime is invisible.

we also ignore Van-der-Waals interactions that are only important at the very edge in the partial wetting regime. Formally we set $A=0$. In this case we minimise over $\left[0, r^{*}\right]$ and find a parabolic profile as for usual liquids:

$$
z(r)=\frac{a_{\|}}{2} r^{2}+b_{\|} r+c_{\|} \quad \text { for } r_{\mathrm{c}} \lesssim r<r^{*}
$$

where $a_{\|}=\frac{m}{2 \bar{\gamma}_{\| \text {air }}}$ and the two constants $b_{\| \mid}$and $c_{\|}$must be chosen so that Young's law holds, i.e., the forces balance, and $z(0)=0$. These conditions lead to $b_{\|}=\left(C / \bar{\gamma}_{\| \cdot \text { arr }}\right)^{1 / 2}$ and $c_{\|}=0$.

Now turning our attention to the second of the two integrals in equation $(7)$, over $\left[r^{*}, \infty\right]$, we assume $\bar{K}_{\perp} / \bar{\gamma}_{\mathrm{B} \text { arr }} \ll 1\left(\bar{K}_{\perp} \simeq \bar{\gamma}_{\mathrm{AB}}\right)$ with the result that the bending contribution $\sim$ $\frac{\bar{K}_{\perp} z}{2}\left(\frac{\mathrm{d}^{2} z}{\mathrm{~d} r^{2}}\right)^{2}$ to the free energy $F_{\perp}(2)$ may be neglected, as can be checked explicitly. The Euler equation then yields

$$
\frac{\mathrm{d} z}{\mathrm{~d} r}=\sqrt{(z+2)(z-1)^{2} / \bar{\gamma}_{\mathrm{B} \cdot \mathrm{arr}}}
$$

where we have used the boundary conditions that as $r \rightarrow \infty$ we require $\frac{\mathrm{d} z}{\mathrm{~d} r} \rightarrow 0$ and $z \rightarrow 1$. Integrating equation (11), we find $r(z)$

$$
r-r^{*}=\sqrt{\frac{\overline{\boldsymbol{\gamma}}_{\mathrm{B} \text { air }}}{3}} \log \left|f\left(z^{*}\right) / f(z)\right| \quad \text { for } r \geq r^{*}
$$


where $f(z)=\frac{\sqrt{(2+z) / 3}-1}{\sqrt{(2+z) / 3}+1}$ and $z\left(r^{*}\right) \equiv z^{*}$. Equation (12) may be inverted to give $z(r)$

$$
z=3\left(\frac{1+\alpha}{1-\alpha}\right)^{2}-2
$$

where $\alpha(r)=f\left(z^{*}\right) \exp \left[-\sqrt{\frac{3}{\bar{\gamma}_{\mathrm{B} \text { alr }}}}\left(r-r^{*}\right)\right]$.

For a complete description we still need to determine $z^{*}, r^{*}$ is then fixed by equation (10). Minimising equation ( 7$), z^{*}$ is given by the solution of

$$
F_{\| 1}\left(z^{*}\right)-F_{\perp}\left(z^{*}\right)=0,
$$

which must be solved numerically if $A$ is non-zero but if we continue to neglect Van-der-Waals terms it may be solved explicitly by substitution of equations (8) and (11) into equations (5) and (2), respectively. In this case $z^{*}$ is given by the physical (smallest, positive) root of the following cubic equation:

$$
z^{* 3}-3 \bar{\gamma}_{\mathrm{AB}}^{2 / 3} z^{*}+\left(2-C / 2-\bar{\gamma}_{I I}\right)=0
$$

Our treatment is self-consistent when the $\|$ region is much larger than the end-to end-distance for individual chains, i.e., $r^{*} \gg 1$. In this case the continuous description is appropriate and also the size of the defect is much smaller than the other length scales of interest. For selfconsistency we must be near the two wetting transitions, for $\perp$ and " chains. To see this consider again the case $A=0$ as we approach the two transitions $m, C \rightarrow 0$ : Expanding equation (10), we find scaling results for $r^{*}$ near the wetting transitions.

$$
r^{*} \simeq \begin{cases}4 \bar{\gamma}_{11 \cdot \text { air }} / m & \text { for } m / C \gg 1 \\ \sqrt{\bar{\gamma}_{\| \cdot \text { air }} / C} & \text { for } m / C \ll 1\end{cases}
$$

Small pieces of monolayer on a surface are not stable with respect to a single piece. There is an "edge penalty" manifested in a line tension $L$. This is obtained by direct integration of the potential $F_{\text {тот }}$ over the volume of the edge $(z<1)$. The energy per unit length of the contact line (in length units of $b$, the monomer size) is

$$
L=\mu\left\{\frac{\sqrt{\bar{\gamma}_{\| \cdot \text { air }}}}{3 m}\left[\frac{\left(C / 2+\bar{\gamma}_{\|}+m z\right)^{2}}{\sqrt{m z+C}}\right]_{0}^{z^{*}}+\frac{4 \sqrt{\bar{\gamma}_{\mathrm{B} \cdot \text { air }}}}{15}\left[(z+2)^{3 / 2}(z-3)\right]_{z^{*}}^{1}\right\}
$$

where $C$ is given by equation (9) if we neglect contributions from the microscopic edge (where bending and Van-der-Waals interactions become significant). We also neglect the contribution from the defect situated between the $\perp$ and is regions.

Finally, we examine the region where Van-der-Waals interactions dominate. This occurs in the very thinnest part of the layer where equation (8) can be approximated by

$$
g(z) \equiv\left(\frac{\mathrm{d} z}{\mathrm{~d} r}\right)^{2}=\frac{A}{4 \pi \bar{\gamma}_{\| \cdot \mathbf{a l r}} z^{2}}
$$

which has the solution

$$
z(r)=\left(\frac{A}{\pi \bar{\gamma}_{\| \cdot \text { alr }}}\right)^{1 / 4} r^{1 / 2},
$$

where we have used the Boundary condition $z(0)=0$. Equation (19) is appropriate provided $4 \pi z^{2}(m z+C) \ll A$.

Some of the main results of this paper are equations (8), (13) and (15), which together give our estimate for the surface contour, and equation (17), which gives the line tension of the edge (minus the defect). 


\section{Bilayer or Monolayer ?}

Up to this point we have assumed that the equilibrium configuration is an AB monolayer, with an energy density $\mu$ from equation (2). We now determine when an ABBA or BAAB bilayer is the equilibrium state. A similar discussion can be found in reference [13]. We identify the equivalence of a bilayer and a monolayer of ABBA units under the natural rescaling $N^{\mathrm{bi}}=2 N$, $\gamma_{A B}^{\text {bi. }}=2 \gamma_{A B}$ and

$$
\tilde{\gamma}^{\text {bi. }}=\left\{\begin{array}{lll}
2 \gamma_{\mathrm{AB}}+\gamma_{\hat{\mathrm{AS}}}+\gamma_{\mathrm{A} \text { alr }} & \text { if } \gamma_{\hat{\mathrm{AS}}}+\gamma_{\mathrm{A} \text { air }}<\gamma_{\hat{\mathrm{BS}}}+\gamma_{\mathrm{B} \cdot \mathrm{aur}} \text { (ABBA bilayer) } \\
2 \gamma_{\mathrm{AB}}+\gamma_{\hat{\mathrm{BS}}}+\gamma_{\mathrm{B} \cdot \mathrm{arr}} & \text { if } \gamma_{\hat{\mathrm{AS}}}+\gamma_{\mathrm{A} \text { air }}>\gamma_{\hat{\mathrm{BS}}}+\gamma_{\mathrm{B} \cdot \mathrm{arr}}
\end{array}\right.
$$

Using this rescaling the energy densities of a bilayer and monolayer are related by equation (2)

$$
F_{\perp}^{\text {bi. }} / F_{\perp}^{\text {mon. }} \equiv \Theta=\left(\frac{\tilde{\gamma}^{\text {bi. }}}{2 \tilde{\gamma}^{\text {mon. }}}\right)^{2 / 3}
$$

with the monolayer therefore more stable if $\Theta<1$ and the bilayer more stable otherwise. The ratio of thicknesses of the bilayer to the monolayer is $2 \sqrt{\Theta}$ and so the bilayer is stable only if its thickness is less than twice that of the monolayer. The discussion of Section 3 above, regarding the wetting transitions for $\perp$ and II chains, still applies when the $\perp$ layer is a bilayer (at $r \rightarrow \infty$ ) provided we rescale the parameters as described above. There may also be region(s) in the copolymer film where the chains are assembled either in a $\perp$ monolayer or as II chains, due to the constraints of the film thickness and interfacial tensions. In fact there are 6 possible architectures for the bilayer near $r=0$, as shown in Figure 3. In order to predict which of these architectures occurs, we might, at the most simple level, calculate the transition points ( $z$-values) between a flat bilayer and I chains, a flat monolayer and I chains and a flat monolayer and bilayer. Setting $\frac{\mathrm{d} z}{\mathrm{~d} r}=0$ we find that the approximate monomer-\| transition values $z^{* \text { mon. }}$ are given by

$$
z^{* \text { mon. } 3}-3 \bar{\gamma}_{\mathrm{AB}}^{2 / 3} z^{* \text { mon. }}+2\left(1-\bar{\gamma}_{I I}\right)=0
$$

the physically interesting roots of this cubic equation are the two largest, the smaller of these is denoted $z_{1}^{* \text { mon. }}$ and the larger $z_{2}^{* \text { mon. }}$. Assuming the monolayer exists at all one real positive root is required on physical grounds since $\|$ chains will always have a lower energy than a monolayer in the limit $z \rightarrow \infty$. If $z_{1}^{* \text { mon. }}>0$, corresponding to $\bar{\gamma}_{\|}<1$, we have a $\|$ region near $z \rightarrow 0$ as discussed in Section 3. The result (22) is identical to equation (15) up to terms $O\left(C^{2}\right)$ and the two forms therefore coinc1de as we approach the wetting transition for the "chains. Simlarly, the one physically important bilayer-" transition value is given by the smallest positive root of

$$
\left(z^{* \text { bi. }} / 2\right)^{3}-3 \bar{\gamma}_{\mathrm{AB}} z^{* \text { bi }} / 2+2\left(\Theta^{3 / 2}-\bar{\gamma}_{I I} / 2\right)=0,
$$

where $z^{\text {bi. }}$ and $z^{* \text { mon. }}$ are both in units of $h_{\infty} \equiv h_{\infty}^{\text {mon. }}$. We can now estimate which of the architectures of Figure 3 occurs: Case (a) (bilayer $\rightarrow \| \rightarrow$ monolayer $\rightarrow \|$ ) occurs when $z^{* \text { bi. }}>z_{2}^{* \text { mon. }}>z_{1}^{* \text { mon. }}>0$; case (b) (bilayer $\rightarrow$ monolayer $\rightarrow \| l$ ) applies when $z_{2}^{* \text { mon. }}>z^{* \text { bi. }}>$ $z_{1}^{* \text { mon. }}>0$; case (c) (bilayer $\rightarrow \| \rightarrow$ monolayer) applies when $z^{* \text { bi. }}>z_{2}^{* \text { mon. }}>0>z_{1}^{* \text { mon. }}$; case (d) (bilayer $\rightarrow$ monolayer) applies when $z_{2}^{* \text { mon. }}>z^{* \text { bi. }}>0>z^{* \text { mon.; }}$ case (e) (bilayer $\rightarrow \|$ ) applies when $z_{1}^{* \text { mon. }}>z^{* \text { bi. }}>0$; case (f) (simple bilayer only) applies when $z_{2}^{* \text { mon. }}<0$ and $z^{* \text { bi. }}<0$. 
(a)

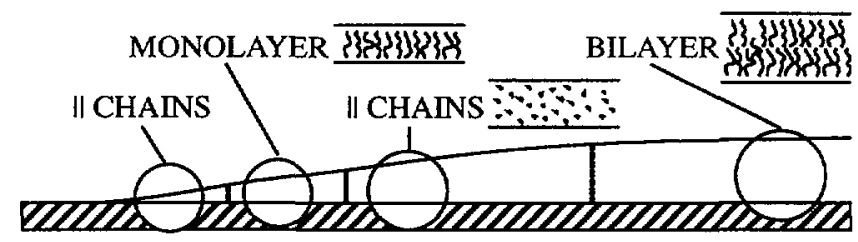

(b)

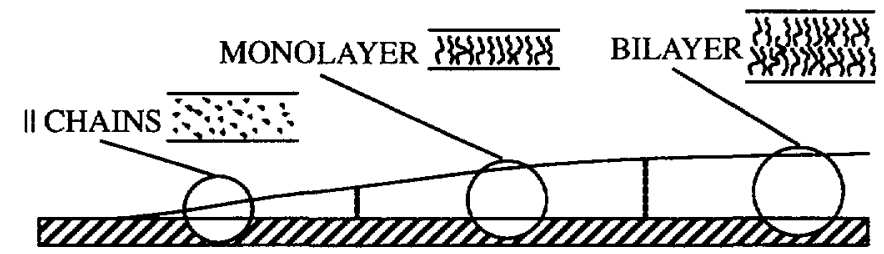

(c)

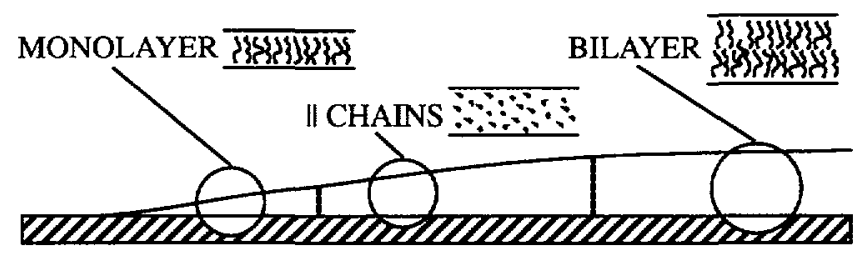

(d)

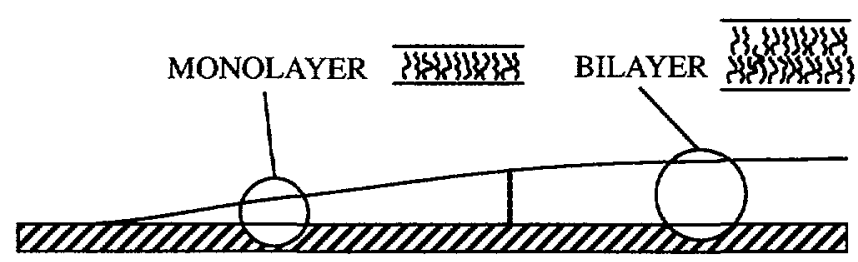

(e)

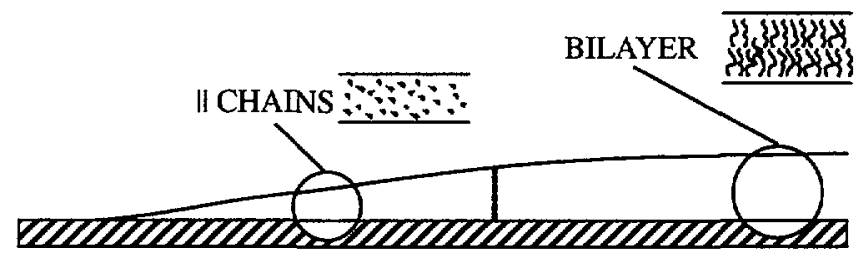

(f)

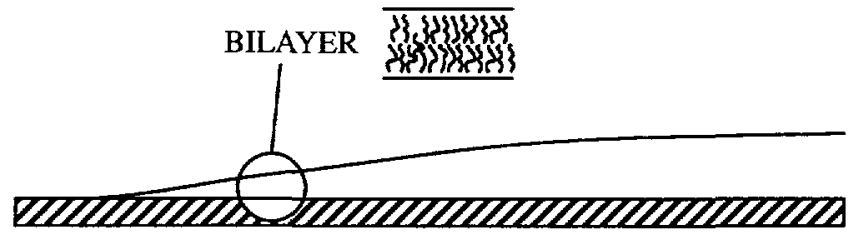

Fig. 3. - The six possible architectures of a wetting bilayer of copolymers near $r=0$. In each case the approximate positions of the defects are given by vertical dashed lines. For decreasing $r$ one sees either: a) bilayer $\rightarrow \| \rightarrow$ monolayer $\rightarrow \|$; b) bilayer $\rightarrow$ monolayer $\rightarrow \|$; c) bilayer $\rightarrow \| \rightarrow$ monolayer; d) bilayer $\rightarrow$ monolayer; e) bilayer $\rightarrow \|$ or $f$ ) simple bilayer only. The conditions under which each of these occurs are discussed in the text. 


\section{Discussion}

Direct experimental observation of a region of $\|$ ordering (e.g., by atomic force machine) may be made possible by the fact that it will present a sequence of strips of $A, B, A \ldots$ at the alr interface. Since these two polymers in general provide different surface tensions at the air interface the surface contour should develop a characteristic ripple with a wavelength of the order of the end-to-end distance of a $\|$ chain, $\bar{\gamma}_{A B}^{1 / 3}$ in $h_{\infty}$ units. However this signature, characteristic of $\|$ ordering, may be difficult to detect, as the amplitude will typically be rather small, at least when both the polymer-air interfacial tensions are large. (This effect involves higher-order corrections to the free energy (4).

Experimentally there are several ways to control the various surface tension parameters. These include variation of the copolymer's chemical components, the ratio of the two block lengths of the copolymer or the composition of either the surface or the third surrounding phase, denoted air in the discussion above. This phase need not be air, it could also be chosen to be a high molecular weight polymer. Variation of the ratio of the block lengths of the copolymer, controlled by the parameter $f \equiv N_{\mathrm{A}} / N$ with $N_{\mathrm{A}}$ the A-block length, allows independent variation of $\gamma_{\| \text {-air }}$ and $\gamma_{\| 1 \text { S }}$. To see this note that in the $\perp$ configuration the same two components are presented at the interfaces, independent of $f$, whereas in the " configuration they are presented in proportions depending on $f$. Hence this may provide one way of studying the $\|$ wetting transition. A first estimate of the surface tensions might be

$$
\gamma_{\| l \cdot S}=f \gamma_{\hat{A} S}+(1-f) \gamma_{\hat{B S S}} \quad \text { and } \quad \gamma_{\| l \cdot a i r}=f \gamma_{\hat{A} \text { air }}+(1-f) \gamma_{\mathrm{B} \cdot \mathrm{alr}}
$$

The present model is based on the assumptions (i) that a continuous representation of the layer is appropriate, and (ii) that the effect of the defect, which occurs at the interface between the "I and $\perp$ regions, may be neglected. These assumptions are reasonable, provided the lateral extent of the $\|$ region is greater than the end-to-end distance of the chains. This is the case provided we are relatively close to the two wetting transitions (see Eq. (16)). When these assumptions fail a more detailed description, including the discrete nature of the individual chains and the internal structure of the defect, becomes necessary. A description of this nature would represent a considerable undertaking and is beyond the scope of the present work.

We now comment on three possible cases where the present model may fail. The first of these is related to the degree of ordering in the copolymer melt. The ODT is found [10] at $\chi N \approx 10$, where $\chi$ is the Flory interaction parameter for the $\mathrm{A}$ and $\mathrm{B}$ blocks. In most experimental samples the chains are not very long $(N \approx 1000$ might be typical) and $\chi N$ is not many times larger than this critical value. Even so it is known that theories constructed in the strong segregation limit [1] often give quite reasonable agreement with experiment. There is also the related question of whether constraining the layer thickness to be less than that found in the bulk can promote disorder. To see this note that for finite $\chi N$ the AB interface is not perfectly sharp but rather the two blocks are significantly mixed over a distance $\Delta$ which is predicted [1] to vary as $\Delta \simeq \chi^{-1 / 2}$ (in monomer units). Thus the layer may become disordered wherever any chain is constrained to have an end to end distance $\lesssim \Delta$. Since the smallest such distance is $z^{*} h_{\infty}$ we require $z^{*} h_{\infty}>\Delta$ for our treatment to be consistant, which is a rather weak restriction.

We also emphasise that finite polydispersity corrections will arise as a consequence of inhomegeneities in the spatial distribution of chain lengths: One might imagine that shorter chains would be concentrated in the $\perp$ region close to the defect.

Finally there is the question of whether free volume plays an important role [20]. Free volume, which may actually be rarefied arr, accounts for any region of reduced density near in a copolymer melt. The effects of free volume may be less if the gas phase (air) is replaced by 
a high molecular weight homopolymer. In the present work we have completely neglected free volume, which is equivalent to taking the limit of large air-copolymer interaction parameters. Whilst the air-copolymer interaction parameter is large, in the sense that there is a large surface tension at the air-copolymer interface, it is not clear that free volume plays no role whatsoever, particularly in the release of constraints in the very thinnest region of the wetting layer. However a complete theory, including the effects of free volume, is beyond the scope of the present work.

\section{Acknowledgments}

The authors would like to acknowledge fruitful discussions with Tom Russel (IBM Almaden). MST would also like to thank Peter Olmsted (Cambridge, UK) and Yun Liu (SUNY, US) for stimulating discussions. Thanks are also extended to the authors of reference [11] for providing a copy of their work prior to publication.

\section{Appendix A}

\section{Films Confined Between Two Solid Surfaces}

In the main body of this paper we have discussed the wetting properties of a copolymer film on a substrate. The case where a copolymer film is confined between two solid surfaces may also be studied within a similar framework. One earlier study [15] considered only polymers which were constrained to be $\perp$ to the two (identical) confining plates. Later, a more general study was also made [14] where the transition to ॥ ordering was also discussed. Some, but not all, of the results presented below can be found in earlier works [13-15] but we nonetheless choose to include them here for completeness.

In this Appendix we present the free energies for a copolymer film in various ordering geometries confined between two flat surfaces, denoted 1 and 2, seperated by a distance $d$. The relevant surface tension parameters, in addition to the inherant $\gamma_{A B}$, are $\gamma_{A 1}=\gamma_{A 1}-\gamma_{1 \text { a1r }}$, $\gamma_{\hat{B} 1}=\gamma_{B 1}-\gamma_{1 \cdot \text { arr }}, \gamma_{\hat{A} 2}=\gamma_{A 2}-\gamma_{2 \text { aar }}$ and $\gamma_{\hat{B} 2}=\gamma_{B 2}-\gamma_{2 \text { arr }}$, where the components and plates are labelled so that $\gamma_{\hat{A 1}}$ is the smallest of these. In what follows one layer is defined to be an entire $A B B A$ repeat unit but the plate seperation need not be an integer or half-odd-integer number of bulk equilibrium layer spacings. We consıder here four different ordering geometries in the strong segregation limit and assume a strong enough propensity for lamellar ordering, i.e., not too close to the phase boundaries of the lamellar region. The first two are "pure" states in which the lamellar orientation doesn't change abruptly anywhere: (a) A stack of layers in which the individual poymers are $\perp$ to the confining plates (the layers are parallel to them). (b) A stack of layers in which the polymers are 1 to the plates (the layer normals are parallel to them). This geometry was also discussed in reference [16]. In addition we consider two "mixed" geometries for which we restrict our attention to plate seperations of at least a few layer spacings. We later demonstrate that these "mixed" geometries never occur. (c) A region of $\perp$ ordering (parallel layers) existing close to surface 1 with a region of $\|$ ordering (perpendicular layers) close to surface 2 . In this case we might think of some planar defect, with a thickness of the order of one layer spacing, between the $\perp$ and I| regions. This is appropriate for plate seperations of a few layers or more (the penetration length in the lamellar (smectic A) phase is of order the layer spacing $[18,21]$ ). (d) Regions of $\perp$ ordering (parallel layers) exist close to both surfaces but a qualitatively different region of, e.g., " ordering (perpendicular layers), exists in the sample interior. In fact we need assume only that this region is not quantised in the direction normal to the plates (in contrast to the $\perp$ phase). As a natural extension of (c) 
one might imagine two planar defects between the one $\|$ and two $\perp$ regions although a single broad defect may be a better description; both lead to the same form for the free energy.

The energies of the four phases described above, per chain, are

$$
\begin{gathered}
F_{a}=F_{\text {bulk }}\left[\left(d^{\prime} / n\right)^{2}+2 n / d^{\prime}+\left\{\gamma_{\hat{A} 1}+\gamma_{\hat{A} 2}+\frac{1-(-1)^{2 n}}{2}\left(\gamma_{\hat{\mathrm{B} 2}}-\gamma_{\hat{\mathrm{A} 2}}\right)\right\} /\left(\gamma_{\mathrm{AB}} d^{\prime}\right)\right] / 3 \\
F_{b}=F_{\mathrm{bulk}}\left[3+\left\{\left(\gamma_{\hat{\mathrm{A} 1}}+\gamma_{\hat{\mathrm{B} 1}}\right) / 2+\left(\gamma_{\hat{\mathrm{A} 2}}+\gamma_{\hat{\mathrm{B}} 2}\right) / 2\right\} /\left(\gamma_{\mathrm{AB}} d^{\prime}\right)\right] / 3 \\
F_{c}=F_{\mathrm{bulk}}\left[3+\left\{\gamma_{\hat{\mathrm{A} 1}}+\left(\gamma_{\hat{\mathrm{A} 2}}+\gamma_{\hat{\mathrm{B} 2}}\right) / 2+\mu_{c}\right\} /\left(\gamma_{\mathrm{AB}} d^{\prime}\right)\right] / 3 \\
F_{d}=F_{\mathrm{bulk}}\left[3+\left\{\gamma_{\hat{\mathrm{A} 1}}+\gamma_{\hat{\mathrm{A} 2}}+\left(\gamma_{\hat{\mathrm{B} 2}}-\gamma_{\hat{\mathrm{A} 2} 2}\right) \theta\left(\gamma_{\hat{\mathrm{A} 2}}-\gamma_{\hat{\mathrm{B} 2}}\right)+\mu_{d}\right\} /\left(\gamma_{\mathrm{AB}} d^{\prime}\right)\right] / 3
\end{gathered}
$$

where $F_{\text {bulk }}=3\left(k \gamma_{\mathrm{AB}}^{2} N\right)^{1 / 3} / 2$ is the bulk energy per chain, $d^{\prime}=d /\left(8 \gamma_{\mathrm{AB}} N^{2} / k\right)^{1 / 3}$ is the plate seperation in units of the bulk ABBA bilayer thickness, $\mu_{c}$ and $\mu_{d}$ can be thought of as "effective surface tensions" associated with the planar defect(s) of $(c)$ and $(d)$ and $n$ is the number of layers present, quantised in units of $1 / 2$ (monolayers). Provided there are several layers present we expect the energetic contribution from the planar $\operatorname{defect}(s)$ to be rather well modelled by a simple surface tension, i.e., with an energy/area independent of the plate seperation. An order of magnitude estimate follows from the fact that $\gamma_{A B}$ is the inherant energy scale $\mu_{c} \simeq \mu_{d} \simeq \gamma_{\mathrm{AB}}$.

We can make several qualitative observations by inspection of equations (A.1)-(A.4): When there is a strong preferential affinity for one of the blocks at each of the surfaces $\perp$ ordering is induced at the surfaces. Even without such strong affinities there are "happy" plate seperations, corresponding to minima of equation (A.1), where there is no frustration and hence no other orientation with a lower energy. However for particularly unhappy plate seperations, near the maxima of equation (A.1), one might imagine the possibility of a "window" in which (b), (c) or (d) become energetically preferable. In fact we show below that (b) can occur but (c) and (d) never do, at least for plate seperations larger than a few layers.

We notice first that states of (a) with $n$ half odd integer can only occur when the plates are different; otherwise $F_{a} \geq F_{b}$ (with the degeneracy $F_{a}=F_{b}$ at certain happy plate seperations). Geometry (a) is always favoured in conditions of strong preferential surface affinity. Whether integer or half odd integer states occur depends on the block selected at each surface. To see if state (b) occurs we compare the energies $F_{b}$ and $F_{a}$. We look first at half odd integer $n$ with $\gamma_{\hat{B} 2}<\gamma_{\hat{A 2}}$, although a similar argument can be constructed for integer $n$, with similar conclusions. For geometry (a) the values of $d^{\prime}$ where $n$ changes, 1 e., where $F_{a}(n)$ and $F_{a}(n+1)$ have the same energy are given by [15]

$$
d^{\prime}=\left[2 n^{2}(n+1)^{2} /(2 n+1)\right]^{1 / 3} .
$$

Using the fact that these values correspond to local maxıma in $F_{a}$ we look first at $n \gg 1$ where the maxima of $\Delta F_{a b}=F_{a}-F_{b}$ are, to leading order in $1 / n$,

$$
\Delta F_{a b}=F_{\mathrm{bulk}}\left[3 /\left(4 n^{2}\right)-(1 / n)\left\{\left(\gamma_{\dot{\mathrm{B} 2}}-\gamma_{\hat{\mathrm{A} 1}}\right) / 2+\left(\gamma_{\hat{\mathrm{A} 2}}-\gamma_{\mathrm{B} 2}\right) / 2\right\} / \gamma_{\mathrm{AB}}\right]
$$

Geometry (b) only occurs when $\Delta F_{a b}>0$, i.e., when $\left\{\left(\gamma_{\hat{\mathrm{B} 1}}-\gamma_{\hat{\mathrm{A} 1}}\right) / 2+\left(\gamma_{\hat{\mathrm{B} 2}}-\gamma_{\hat{\mathrm{A} 2}}\right) / 2\right\} / \gamma_{\mathrm{AB}}<$ $3 /(4 n) \ll 1$. Thus as the plate seperation is increased there is an incresingly restrictive condition on the surface tension differences which must be satisfied for geometry (b) to be preferred. In the opposite limit $d \ll 1 / 2 \|$ ordering occurs when $\left\{\left(\gamma_{\hat{B} 1}-\gamma_{\hat{A 1}}\right) / 2+\left(\gamma_{\hat{A} 2}-\right.\right.$ $\left.\left.\gamma_{\hat{\mathrm{B} 2}}\right) / 2\right\} / \gamma_{\mathrm{AB}}<1$, which can be thought of as a simple wetting condition. These two limits $d \gg 1, d \ll 1$ can be thought of as giving upper and lower bounds for the inequality governing the existence of $(b)$. 
Within this scheme we can only discuss the existence of (c) and (d) in the large $n$ limit where the size of the defect region(s) is much smaller than $d$. In this limit we compare (c) and (d) with (a) (say). Following the same arguments as above the maxima of $\Delta F_{a d}=F_{a}-F_{d}$ are, to leading order in $1 / n$,

$$
\Delta F_{a d}=F_{\text {bulk }}\left[3 /\left(4 n^{2}\right)-(1 / n)\left\{\left(\gamma_{\dot{\mathrm{B} 1}}-\gamma_{\dot{\mathrm{A} 1}}\right) / 2+\left(\gamma_{\hat{\mathrm{A} 2}}-\gamma_{\overrightarrow{\mathrm{B} 2}}\right) / 2+\mu_{d}\right\} / \gamma_{\mathrm{AB}}\right]
$$

Since $\mu_{d} / \gamma_{\mathrm{AB}} \simeq 1$ we see immediately that $\Delta F_{a d}<0$ and so (d) cannot occur. A similar argument leads to the same conclusion for (c).

These results makes sense if one understands that the lamellar stack becomes softer as it becomes larger: The frustration energy (per chain) associated with an unfavourable number of layers in (a) decays as $1 / n^{2}$ whilst the surface energy penalty for (b) only decays as $1 / n$. By choosing a small enough prefactor (the surface tension difference appearing in Eq. (A.6)) one can still match these terms. However this is not the case for (c) or (d) as the prefactor to the surface energy penalty has a lower bound of order unity and so (c) and (d) are always metastable with respect to (a).

The preceding model seems to be the simplest which allows for a comparison of the four ordering geometries mentioned. More complex ordering may occur whenever we approach either (i) the lamellar phase boundaries, (ii) small plate seperations or (iii) the weak segregation limit (higher temperatures). As a final point it is perhaps worth mentioning that the relaxation times for re-orientation between different ordering geometries may be rather long.

\section{Appendix B}

\section{Results for Parallel Chains Anchored in the $\| \nabla$ Orientation}

In this Appendix we consider chains permanently anchored in the $\|, \nabla$ orientation where the chain end-to-end vectors point towards the wetting edge (along the $\nabla z$-direction) and lie roughly in the plane of the substrate. Such anchoring could, in principle, occur at the surface of the substrate. It is also possible that the defect may impose an ordering direction on the chains nearby.

In determining the free energy $F_{\|, \nabla}$ analogous to that given in equation (5) we include the leading order term in a gradient expansion at the polymer-air interface. The bending contribution $\frac{\kappa_{\|}}{2 h^{2}}\left(\frac{\mathrm{d} h}{\mathrm{~d} x}\right)^{2}$ to leading order, scales like the squared angle between the end-toend vector of neighbouring chains $\theta \simeq \frac{1}{h} \frac{\mathrm{d} h}{\mathrm{~d} x}$ with a bending modulus estimated by $\kappa_{\|} \simeq \gamma_{\mathrm{AB}} y$.

$$
F_{\|, \nabla}=\mu\left\{\bar{\gamma}_{\mathrm{AB}}^{2 / 3}+\frac{2}{3 z}\left(\bar{\gamma}_{\|}+\left(\frac{\bar{\gamma}_{\| \cdot \mathrm{air}}}{2}+\frac{\bar{K}_{\|}}{2 z}\right) \cdot\left(\frac{\mathrm{d} z}{\mathrm{~d} r}\right)^{2}\right)+\frac{A}{12 \pi z^{3}}\right\}
$$

where $\bar{K}_{\|}=\bar{\gamma}_{\mathrm{AB}}^{4 / 3}$.

This new free energy results in changes to the treatment of the surface contour near the edge of the wetting layer (Section 4 above) as we will now discuss.

We construct the total free energy $F_{\text {тот }}$ of the layer, analagous to equation (7), as the sum of the contributions from the $\perp$ and $\|, \nabla$ regions and consider first the integral $\int_{0}^{r^{*}}\left(F_{\|, \nabla}-\mu\right) z \mathrm{~d} z$. Direct minmisation gives $g(z) \equiv(\mathrm{d} z / \mathrm{d} r)^{2}$

$$
g(z)=\frac{m z+A /\left(4 \pi z^{2}\right)+C}{\bar{K}_{\|} / z+\bar{\gamma}_{\| \cdot \mathrm{air}}},
$$


where the integration constant $C$ is fixed by Young's law, as before equation (9). In the following we ignore Van-der-Waals interactions and proceed by dividing the free energy integral into two regions $\left[0, r_{\mathrm{c}}\right]$ and $\left[r_{\mathrm{c}}, r^{*}\right]$ where we shall find that the contribution due to bending $\sim \frac{\bar{K}_{\|}}{z}\left(\frac{\mathrm{d} z}{\mathrm{~d} r}\right)^{2}$ in equation (7) is negligible for $r_{\mathrm{c}} \ll r<r^{*}\left(z_{\mathrm{c}} \equiv z\left(r_{\mathrm{c}}\right) \ll z\right)$. For simplicity we will merely employ the two asymptotic regimes for $r>r_{\mathrm{c}}$ and $r<r_{\mathrm{c}}$.

Neglecting the bending contribution and minimising over $\left[r_{c}, r^{*}\right]$ we find a parabolic profile the same as that given in equation (10). If $\bar{K}_{\|}=0$ and the bending term is absent we simply have $b_{\|}=\left(C / \bar{\gamma}_{\| \cdot \text { air }}\right)^{1 / 2}$ and $c_{\|}=0$ as before.

By examining the region in which this solution (10) is self-consistent we find that the bending contribution is negligible for $z \gg z_{\mathrm{c}}$

$$
z_{\mathrm{c}} \equiv z\left(r_{\mathrm{c}}\right) \simeq \bar{K}_{\|} / \bar{\gamma}_{\| \cdot \mathrm{alr}}
$$

where we have assumed that $\bar{K}_{\| l} / \bar{\gamma}_{\| \cdot \text { air }} \ll 1$, a condition which is almost always true for copolymers for which $\bar{\gamma}_{\mathrm{AB}} / \bar{\gamma}_{11 \cdot \text { air }} \lesssim 1 / 10$ (recalling $\bar{K}_{\| 1} \simeq \bar{\gamma}_{\mathrm{AB}}^{4 / 3}$ and $\bar{\gamma}_{\mathrm{AB}}<1$ ).

We next examine the region $z \ll z_{\mathrm{c}}$ where bending corrections may be important. In this limit, with $A=0$, as before, we have

$$
g(z)=\frac{m z^{2}+C z}{\bar{K}_{\|}}
$$

where we recal the definition $m \equiv 3\left(\bar{\gamma}_{\mathrm{AB}}^{2 / 3}-1\right)$. Equation (B.4) can be solved to give

$$
z(r)=\frac{C}{m} \sinh ^{2}\left[\left(\frac{m}{4 \bar{K}_{\|}}\right)^{1 / 2} r+q\right]
$$

where a sutable boundary condition for the constant $q$ would be continuity of the profile. In this discussion we neglect Van-der-Waals interactions, with the boundary condition $z(0)=0$ and hence $q=0$. We notice immediately from equation (B.5) that the curve has $z \sim r^{2}$ asymptotic behaviour near $z=0$ and hence an apparently vanishing contact angle. However the asymptotic contact angle $\Psi \approx b_{\| 1}$, appearing in equation (10), is still well defined provided we consider the surface contour just outside the regime where the bending corrections become important. In fact at even smaller scales Van-der-Waals interactions impose an infinite slope, as shown by the exact solution (B.2). The $z \sim r^{2}$ scaling can also be identified by simply requiring the contribution to the free energy due to bending $\sim \frac{1}{z}\left(\frac{\mathrm{d} z}{\mathrm{~d} r}\right)^{2}$ to remain finite as we approach the edge. The value $r_{c}$ is obtained by inversion of equation (B.5)

$$
r_{\mathrm{c}}=2\left(\bar{K}_{\|} / m\right)^{1 / 2} \sinh ^{-1}\left(m z_{c} / C\right)^{1 / 2}
$$

Using the value $z_{\mathrm{c}}=\bar{K}_{\| l} / \bar{\gamma}_{\| 1 \cdot \text { alr }}$ we find the following asymptotic results for $r_{\mathrm{c}}$

$$
r_{\mathrm{c}}=\left\{\begin{array}{ll}
\frac{2 \bar{K}_{\|}}{\sqrt{C} \bar{\gamma}_{\| \text {air }}} & \text { for } \frac{m \bar{K}_{\|}}{C \bar{\gamma}_{\| \text {air }}} \ll 1 \\
\sqrt{\frac{\bar{K}_{\| 1}}{m} \log \frac{4 m \bar{K}_{\|}}{C \bar{\gamma}_{\| \text {air }}}} & \text { for } \frac{m \bar{K}_{\| I}}{C \bar{\gamma}_{\| \text {air }}} \gg 1
\end{array},\right.
$$

which diverges as we approach the two wetting transitions $\bar{\gamma}_{\mathrm{AB}} \rightarrow 1(m \rightarrow 0)$ and $C \rightarrow 0$.

Finally, in the region where Van-der-Waals interactions dominate, equation (B.2) can be approximated by

$$
g(z) \equiv\left(\frac{\mathrm{d} z}{\mathrm{~d} r}\right)^{2}=\frac{A}{4 \pi \bar{K}_{\|} z}
$$


which has a qualitatively different solution to equation (19).

$$
z(r)=(3 / 2)^{2 / 3}\left(\frac{A}{4 \pi \bar{K}_{\|}}\right)^{1 / 3} r^{2 / 3}
$$

\section{References}

[1] Semenov A.N., Sov. Phys.- JETP (Eng. Transl.) 61 (1985) 733.

[2] Leibler L., Macromolec. 13 (1980) 1602.

[3] Hasegawa H. and Hashimoto T., Polymer 33 (1992) 475; Hasegawa H., Tanaka H., Yamasaki K. and Hashimoto T., Macromolec. 20 (1987) 1651.

[4] Almdal K., Koppi K.A., Bates F.S. and Mortensen K., Macromolec. 25 (1992) 1743.

[5] Herman D.S., Kinning D.J., Thomas E.L. and Fetters L.J., Macromolec, 20 (1987) 2940.

[6] Coulon G., Russell, T.P., Deline V.R. and Green P.F., Macromolec. 22 (1989) 2581; Russell T.P., Coulon G., Deline V.R. and Miller D.C., Macromolec. 22 (1989) 4600.

[7] Coulon G., Collin B., Ausserré D., Chatenay D. and Russell T.P. J. Phys. France 51 (1990) 2801; Maaloum M., Ausserré D., Chatenay D., Coulon G. and Gallot Y., Phys. Rev. Lett. 68 (1992) 1575.

[8] Russell T. P., Menelle A., Anastasiadis S. H., Satija S. K. and Majkrzak C. F., Macromolec. 24 (1991) 6263.

[9] Lambooy P., Russell T. P., Kellogg G. J., Mayes A. M., Gallagher P. D. and Satija S. K., Phys. Rev. Lett. 72 (1994) 2899.

[10] Bates F.S. and Fredrickson G.H., Ann. Rev. Phys. Chem. 41 (1990) 525.

[11] Carvalho B.L. and Thomas E.L., preprint, August 1994.

[12] Liu Y., Rafailovich M.H., Sokolov J., Kramer E.J, and Bahal S., to be submitted to Macromolec.

[13] Ausserré D., Raghunathan V.A. and Maaloum M., J. Phys. II France 3 (1993) 1485.

[14] Walton D.G., Kellogg G.J., Mayes A.M., Lambooy P. and Russell T.P., Macromolec. 27 (1994) 6225.

[15] Turner M.S., Phys. Rev. Lett. 69 (1992) 1788. In this reference the symbol $\gamma_{\text {AS }}$ should be identified with the effective surface tension $\gamma_{A-S}$ (similarly for $\gamma_{B S}$ ).

[16] Kikuchi M. and Binder K., J. Chem. Phys. 101 (1994) 3367.

[17] Leger L. and Joanny J.-F., Rep. Progr. Phys. 55 (1992) 431.

[18] Turner M.S. and Joanny J.-F., Macromolec. 25 (1992) 6681.

[19] The bending term is negligible even if we choose $\kappa_{\perp} \simeq \gamma_{\mathrm{AB}} h_{\infty}$, with $h_{\infty}$ the equilibrium (flat) monolayer thickness.

[20] Reiter G., Europhys. Lett. 23 (1993) 579.

[21] de Gennes P.-G. and Prost J., The Physics of Liqud Crystals (Clarendon, Oxford, 1993). 